\title{
Cross-national variations in reported discrimination among people treated for major depression worldwide: the ASPEN/INDIGO international study
}

Antonio Lasalvia, Tine Van Bortel, Chiara Bonetto, Geetha Jayaram, Jaap van Weeghel, Silvia Zoppei, Lee Knifton, Neil Quinn, Kristian Wahlbeck, Doriana Cristofalo, Mariangela Lanfredi, Norman Sartorius, Graham Thornicroft and the ASPEN/INDIGO Study Group

\section{Background}

No study has so far explored differences in discrimination reported by people with major depressive disorder (MDD) across countries and cultures.
Aims
To (a) compare reported discrimination across different countries, and (b) explore the relative weight of individual and contextual factors in explaining levels of reported discrimination in people with MDD.

\section{Method}
Cross-sectional multisite international survey (34 countries worldwide) of 1082 people with MDD. Experienced and anticipated discrimination were assessed by the Discrimination and Stigma Scale (DISC). Countries were classified according to their rating on the Human Development Index (HDI). Multilevel negative binomial and Poisson models were used.

\section{Results}

People living in 'very high HDI' countries reported higher discrimination than those in 'medium/low HDI' countries. variation in reported discrimination across countries was only partially explained by individual-level variables. The contribution of country-level variables was significant for anticipated discrimination only.

\section{Conclusions}

Contextual factors play an important role in anticipated discrimination. Country-specific interventions should be implemented to prevent discrimination towards people with MDD.

\section{Declaration of interest \\ None.}

\section{Copyright and usage}

(c) The Royal College of Psychiatrists 2015.
Major depression is the second leading cause of global disability in the world and the eleventh leading cause of global burden. ${ }^{1}$ Although a number of effective treatments are currently available, fewer than half of people with depression worldwide receive adequate care. ${ }^{2}$ Among barriers to treatment, stigma and discrimination related to mental disorders play a crucial role. ${ }^{3}$ Mental ill health discrimination is universally experienced and influences many aspects of people's lives: it represents a barrier to social integration, it limits life opportunities and has a negative impact on help-seeking behaviour; it produces changes in feelings and attitudes for both patients (lower self-esteem, poorer self-care and social withdrawal) and their family members (feelings of guilt, shame, despair). ${ }^{4}$ Although the literature shows that participants in studies across the world express largely similar types of expectations and experiences of mental ill health discrimination, some cultural specificities do exist. ${ }^{5,6}$ Empirical evidence demonstrates differences in symptom expression and understanding of illness, and cultural influences have been noted in care-seeking and public acceptance of the illness. ${ }^{7,8}$ However, findings from studies in different cultural contexts are difficult to compare, since research in this field has been conducted by using inconsistent and/or heterogeneous methodologies. Moreover, a considerable amount of information comes from research conducted among ethnic minorities living in Western countries, such as the USA ${ }^{9-11}$ or the $\mathrm{UK},{ }^{12}$ rather than on populations living in their own countries. In addition, cross-cultural research has mainly addressed stigma related to schizophrenia ${ }^{13,14}$ or mental disorders in general, ${ }^{15,16}$ rather than depression specifically. Still, a few studies have shown that this latter condition has better public acceptance than schizophrenia; ${ }^{6,17,18}$ it could therefore be expected that some specificities in depression-related stigma may exist.
Further, cross-cultural research in this field has generally focused on attitudes of the general population towards mental disorders or people with mental disorders, ${ }^{10,16,19,20}$ rather than on the ways in which behavioural consequences of stigma (discrimination) are experienced by people with depression. ${ }^{21}$ Finally, the few existing cross-national comparative studies on stigma and discrimination related to depression have been carried out in Western countries only. $^{22-24}$ To fill these knowledge gaps, the present explanatory study was undertaken and aims, for people with major depressive disorder (MDD), to (a) compare reported discrimination across different countries in the world; and (b) explore the relative weight of individual and contextual factors in explaining levels of reported discrimination.

\section{Method}

\section{Design}

Data were collected within the frame of the European Union (EU)-funded ASPEN (Anti Stigma Programme European Network) study, which was nested within the larger INDIGODepression (International Study of Discrimination and Stigma for Depression) research network. Full details of the ASPEN/ INDIGO-depression study are given elsewhere. ${ }^{25}$ Overall, 40 sites in 34 countries worldwide were included (Africa: Egypt, Morocco, Nigeria (4 sites), Tunisia; America: Brazil, Canada, Venezuela; Asia: India, Japan, Malaysia, Pakistan, Sri Lanka, Taiwan; Europe: Belgium, Bulgaria, Croatia, Czech Republic, Finland, France, Germany, Greece, Hungary, Italy (2 sites), Lithuania, The Netherlands, Portugal, Romania, Serbia, Slovakia, Slovenia, Spain, Turkey, UK (2 sites); Oceania: Australia). 


\section{Participants}

This was intentionally a pragmatic study ${ }^{25}$ in which many lowand middle-income countries were included who participated using only locally available resources as no external grant provision was available. Within centres, site directors were asked to identify a minimum of 25 participants who were, in their judgement, reasonably representative (as a group) of all people with a diagnosis of MDD attending specialist mental health services (either out-patient or day care in both the public and private sectors in the local area). The minimum number of 25 for each site was defined for feasibility issues, particularly for non-European sites with no grant support. This method, used in our previous schizophrenia study, ${ }^{26}$ was intended to allow local staff to take into account the specific local service configuration and to draw participants from the whole range of appropriate local services. Staff at each site ensured that the sample had a spread across the adult age range (young people (18-25 years), working years $(25-65)$ and older adults $(\geqslant 65)$ ) and a clear representation of female participants as MDD is twice as prevalent in women as men. Inclusion criteria were: (a) clinical diagnosis of MDD (single episode or recurrent) according to DSM-IV-TR ${ }^{27}$ criteria during the previous 12 months (diagnosis had been made by patients' treating clinicians at the time of treatment contact and was not reassessed at the time of study recruitment); (b) written informed consent to participate; (c) ability to understand and speak the main local language; and (d) aged 18 or older. Exclusion criteria were: (a) being a psychiatric in-patient at time of recruitment; and (b) having a comorbid diagnosis of schizophrenia (other comorbidities were accepted). The ASPEN/INDIGOdepression study was approved by the appropriate ethical review board at each study site.

\section{Measures}

Participants were assessed face-to-face by independent researchers not involved in the care process using the Discrimination and Stigma Scale (version 12; DISC-12) ${ }^{28}$ - a structured mix-method interview for recording the discrimination experienced by an individual with a mental disorder. Full details of the psychometric properties of this scale are reported elsewhere. ${ }^{28}$ DISC-12 asks 32 questions about aspects of everyday life including work, marriage, parenting, housing, leisure and religious activities. Items 1-21 are to ascertain experienced discrimination (for example 'Have you been treated unfairly in making or keeping friends?'); items 2225, anticipated discrimination (for example 'Have you stopped yourself from applying for work?'); items 26 and 27, positive treatment (for example, 'Have you been treated more positively by your family?'); and items $28-32$, coping strategies to overcome discrimination (for example 'Have you been able to use your personal skills or abilities in coping with stigma and discrimination?'). Participants' responses were rated with a four-point Likert scale ( 0 , no difference; 1 , a little different; 2 , moderately different; and 3 , a lot different). The DISC-12 items were divided into four subscales - experienced discrimination (0-21), anticipated discrimination (0-4), overcome discrimination and positive treatment. Only the first two subscales will be addressed here. For each subscale a total score is generated by counting the number of items in which participants score 1,2 or 3 . DISC-12 also allows qualitative information to be gathered to add detail to the experiences rated, providing a strong validation for the occurrence, direction and severity of the discrimination rated quantitatively, which is not discussed in this report but will be reported on elsewhere. Sociodemographic and clinical information (years since first contact with mental health services, type of mental healthcare, lifetime number of depressive episodes, admission to psychiatric hospital, advantage of having received a diagnosis of MDD as an explanation for one's own mental health problems) are also recorded.

\section{Stratification of ASPEN/INDIGO study sites}

The ASPEN/INDIGO-depression countries were grouped using the Human Development Index (HDI), ${ }^{29}$ a summary measure of human development produced by the United Nations (for this study we used 2010 data). ${ }^{29}$ The HDI measures the average achievements of a country in three basic dimensions of human development: (a) a long and healthy life; (b) access to knowledge; (c) a decent standard of living. The three dimensions are measured by: (a) life expectancy at birth (data source: UNDESA United Nations Department of Economic and Social Affairs 2009); (b) mean years of schooling (data sources: population censuses and household survey data compiled by UNESCO, EUROSTAT and others) and expected years of schooling (data source: UNESCO Institute for Statistics 2010); (c) gross national income per capita (data source: World Bank 2010 and International Monetary Fund 2010). ${ }^{29}$ Countries are ranked by their HDI rating. HDI classification is based on quartiles and denoted as 'very high', 'high', 'medium' and 'low'. ASPEN/INDIGOdepression participating countries were grouped as follows: 'very high HDI' (Australia, The Netherlands, Canada, Germany, Japan, France, Finland, Belgium, Spain, Greece, Italy, UK, Czech Republic, Slovenia, Slovakia, Hungary, Portugal), 'high HDI' (Lithuania, Romania, Croatia, Malaysia, Bulgaria, Serbia, Brazil, Venezuela, Tunisia, Turkey) and 'medium/low HDI' (Sri Lanka, Egypt, Morocco, India, Nigeria, Pakistan, Taiwan). The categories 'medium' and 'low' were put together as Nigeria was the only 'low HDI' country.

\section{Statistical analysis}

Analyses were performed using Stata 13.0 for Windows. All $P$-values were two-tailed with an accepted significance level of 0.05 . Categorical variables were presented as percentages, and continuous variables were presented as mean values with standard deviations. Comparisons among independent groups were performed by chi-squared and Kruskal-Wallis tests respectively. As a result of the data dependencies induced by the nesting of patients (level 1) within countries (level 2), multilevel models were used, which allowed the simultaneous examination of the effects of individual-level and country-level variables on individual-level outcomes. More specifically, given the distribution of data, negative binomial ('menbreg' command) was suitable for 'experienced discrimination' subscore and poisson ('mepoisson' command) for 'anticipated discrimination' subscore.

The steps in the construction of the models were: (a) study differences in reported discrimination among countries $\left(M_{0}\right.$ : a multilevel model with the country effects modelled as random or intercept only model); (b) include individual variables $\left(M_{1}\right.$ : experienced or anticipated discrimination subscore, age, gender, marital status, working condition, education, advantage to have an MDD diagnosis as an explanation for one's own mental health problems, lifetime number of depressive episodes, out-patient mental healthcare, admission to psychiatric hospital) as fixed effects ('years since first contact with mental health services' was not included because it was significantly associated with 'lifetime number of episodes of major depression'); and (c) include human development classification for country level $\left(M_{2}: M_{1}+\mathrm{HDI}\right)$. Cross-level interactions terms were added where appropriate.

The multivariate models $M_{1}$ and $M_{2}$ were estimated, introducing as patients' characteristics only those variables that were 
found significantly associated $(P<0.05)$ with the dependent variable in the univariate multilevel models. The proportional change in variance estimates of the different models was calculated. This indicates the part that patients' characteristics and countries' HDI classification, respectively, explain concerning the total inter-country variation.

\section{Results}

Overall, 1087 people with MDD worldwide participated in the study. Participants' characteristics stratified by the three HDI country groupings are shown in Table 1 . Country groups differed for all the sociodemographics considered (with exception of gender composition), with participants from the 'medium/low HDI' group showing lower mean age, lower educational level, higher unpaid work rate and lower retired rate. Moreover, country groups differed for almost all the clinical characteristics considered (with the exception of out-patient mental healthcare and advantage to have an MDD diagnosis), with participants from the 'medium/low HDI' group showing lower contact duration with mental health services, lower number of depressive episodes and lower hospital admission rates.

Average scores for experienced discrimination by HDI group were 3.97 (s.d. =3.50) for the 'very high', 3.38 (s.d.=3.34) for the 'high' and 3.30 (s.d.=3.74) for the 'medium/low' group (Fig. 1 ). Average scores for anticipated discrimination by HDI group were $1.68($ s.d. $=1.12)$ for the 'very high', $1.56($ s.d. = 1.13) for the 'high' and 1.24 (s.d.=1.08) for the 'medium/low' group (Fig. 2). The comparison of average scores among the three country groupings revealed significant between-group variation for both experienced (Kruskal-Wallis, $P<0.001$ ) and anticipated (Kurskal-Wallis, $P<0.001$ ) discrimination.

Table 2 shows the comparison of the percentage of participants that endorsed experienced discrimination and anticipated discrimination in the various DISC-12 items among the three country groupings. Experiences of discrimination were more frequent in participants living in 'very high HDI' countries than in 'medium/low HDI' countries; this difference reached statistical significance in most life domains, such as making or keeping friends, family, finding a job, welfare benefits, physical health, mental health staff and parental role (chi-squared, $P<0.05$ ). It should also be noted that the percentage of people reporting to have been shunned or avoided by others is 1.6-fold higher in 'very high HDI' countries than in 'medium/low HDI' countries, which is a highly significant difference (chi-squared, $P<0.001$ ). Only religious practices showed a reverse pattern, with a higher percentage of discrimination reported in the 'medium/low HDI' group. Participants of 'very high HDI' countries also reported more anticipated discrimination than those living in the other two country groups for all the DISC-12 domains (chi-squared, $P<0.05$ ).

Table 3 shows fixed and random parameters estimated from multilevel negative binomial models for experienced discrimination. A significant variation across countries was found (model $M_{0}$, country-level variability not explained by other variables: 0.13 , likelihood ratio test $P<0.001$ ). When individual-level variables were included (model $M_{1}$ ), anticipated discrimination, sociodemographics (age; widowed/separated/divorced marital status; unpaid work, unemployment or student) and clinical characteristics (previous admissions for psychiatric treatment; number of episodes of depression) were statistically significant and random variation between countries decreased by $30.8 \%$ (thus indicating that nearly a third of country-level variation came from differences in the population composition in each country). Stratification of countries according to HDI (model $M_{2}$ ) was not statistically significant and consequently the between-countries variation did not change.

Table 4 shows fixed and random parameters estimated from multilevel Poisson models for anticipated discrimination. A significant variation across countries was found (model $M_{0}$, likelihood ratio test $P<0.001$ ). When individual-level variables were included (model $M_{1}$ ), experienced discrimination, age and single/non-cohabiting partner marital status were statistically significant and random variation between countries decreased by $37.5 \%$. When country stratification according to the HDI was added (model $\mathrm{M}_{2}$ ), the between-countries variation decreased to $62.5 \%$ (thus indicating that $25 \%$ of the country-level variation came from differences in human development). No significant

Table 1 Sociodemographic and illness-related characteristics of the overall sample stratified by country groups (very high Human Development Index (HDI), high HDI, medium/low HDI) $(n=1087)$

\begin{tabular}{|c|c|c|c|c|}
\hline & $\begin{array}{l}\text { Very high HDI } \\
\quad(n=503)\end{array}$ & $\begin{array}{l}\text { High HDI } \\
(n=314)\end{array}$ & $\begin{array}{l}\text { Medium/low HDI } \\
\qquad(n=270)\end{array}$ & $P^{\mathrm{a}}$ \\
\hline Male, \% & 34.0 & 30.3 & 38.5 & 0.110 \\
\hline Age, years: mean (s.d.) & $47.2(15.3)$ & $45.3(14.6)$ & $39.8(14.0)$ & $<0.001$ \\
\hline Lower education, \% & 44.1 & 35.1 & 55.0 & $<0.001$ \\
\hline \multicolumn{5}{|l|}{ Marital status, \% } \\
\hline Married/cohabiting & 44.4 & 51.6 & 59.0 & 0.001 \\
\hline Single & 30.5 & 24.0 & 26.1 & \\
\hline Widowed/separated/divorced & 25.1 & 24.4 & 14.9 & \\
\hline \multicolumn{5}{|l|}{ Employment, ${ }^{\mathrm{b}} \%$} \\
\hline Full time/part time & 39.2 & 39.9 & 40.5 & $<0.001$ \\
\hline Volunteer/sheltered/at home & 4.4 & 8.6 & 22.7 & \\
\hline Unemployed/student & 36.5 & 33.9 & 31.5 & \\
\hline Retired & 19.9 & 17.6 & 5.3 & \\
\hline Years since first mental health contact, mean (s.d.) & $11.7(12.2)$ & $9.2(9.4)$ & $6.4(8.7)$ & $<0.001$ \\
\hline Out-patient mental healthcare, \% & 87.2 & 82.6 & 84.3 & 0.185 \\
\hline $\begin{array}{l}\text { Advantage to have a diagnosis of major depressive disorder } \\
\text { as an explanation for one's own mental health problems, \% }\end{array}$ & 80.8 & 79.0 & 78.6 & 0.737 \\
\hline Lifetime depressive episodes $\geqslant 6, \%$ & 41.4 & 30.0 & 25.0 & $<0.001$ \\
\hline Ever psychiatric admission, \% & 41.2 & 42.4 & 27.2 & $<0.001$ \\
\hline
\end{tabular}


Table 2 Comparison of percentages of participants with major depressive disorder reporting discrimination ${ }^{\mathrm{a}}$ in the various Discrimination and Stigma Scale (DISC-12) items by country groups (very high Human Development Index (HDI), high HDI, medium/low HDI)

\begin{tabular}{|c|c|c|c|c|c|c|c|}
\hline & \multicolumn{2}{|c|}{ Very high HDI $(n=501)$} & \multicolumn{2}{|c|}{ High HDI, $(n=312)$} & \multicolumn{2}{|c|}{ Medium/low HDI, $(n=269)$} & \multirow[b]{2}{*}{$\begin{array}{c}\text { Chi-squarec } \\
P\end{array}$} \\
\hline & $\begin{array}{c}\text { Discriminated } \\
\%\end{array}$ & $\begin{array}{c}\text { Not applicable } \\
\%\end{array}$ & $\begin{array}{c}\text { Discriminated } \\
\%\end{array}$ & $\begin{array}{c}\text { Not applicable } \\
\%\end{array}$ & $\begin{array}{l}\text { Discriminated } \\
\%\end{array}$ & $\begin{array}{c}\text { Not applicable } \\
\%\end{array}$ & \\
\hline \multicolumn{8}{|l|}{ Experienced discrimination $(n=1082)$} \\
\hline Making or keeping friends & 41.1 & 6.2 & 30.4 & 7.3 & 27.8 & 1.9 & $<0.001$ \\
\hline Neighbourhood & 17.2 & 29.6 & 23.2 & 12.7 & 17.8 & 1.5 & 0.116 \\
\hline Dating or intimate relationships & 32.5 & 29.6 & 27.3 & 59.6 & 28.0 & 18.9 & 0.348 \\
\hline Housing & 13.2 & 36.2 & 14.0 & 35.7 & 13.9 & 17.0 & 0.953 \\
\hline Education & 22.7 & 44.3 & 18.6 & 44.6 & 19.5 & 25.6 & 0.511 \\
\hline Marriage or divorce & 38.9 & 35.4 & 30.6 & 28.3 & 31.2 & 23.7 & 0.081 \\
\hline Family & 44.4 & 2.4 & 43.8 & 1.9 & 30.0 & 0.7 & $<0.001$ \\
\hline Finding a job & 27.5 & 46.1 & 23.8 & 45.9 & 16.5 & 29.6 & 0.022 \\
\hline Keeping a job & 34.5 & 29.8 & 31.1 & 32.8 & 26.0 & 36.7 & 0.150 \\
\hline Public transport & 7.7 & 21.9 & 6.4 & 24.5 & 8.8 & 11.1 & 0.607 \\
\hline Welfare benefits or disability pensions & 27.8 & 50.1 & 18.0 & 48.1 & 9.4 & 67.8 & 0.001 \\
\hline Religious practices & 7.2 & 39.0 & 2.7 & 27.1 & 10.1 & 4.4 & 0.005 \\
\hline Social life & 23.1 & 9.9 & 17.0 & 18.8 & 19.7 & 1.5 & 0.149 \\
\hline Police & 11.1 & 40.4 & 5.4 & 28.7 & 9.6 & 22.6 & 0.073 \\
\hline Physical health problems & 23.1 & 6.6 & 15.1 & 4.1 & 11.8 & 2.6 & $<0.001$ \\
\hline Mental health staff & 26.0 & 3.6 & 16.8 & 1.0 & 12.5 & 1.5 & $<0.001$ \\
\hline Personal privacy & 14.3 & 3.4 & 20.7 & 5.4 & 19.4 & 2.2 & 0.044 \\
\hline Personal safety and security & 24.2 & 7.6 & 19.6 & 1.9 & 21.9 & 1.5 & 0.323 \\
\hline Starting a family or having children & 16.8 & 59.4 & 17.0 & 50.6 & 12.7 & 32.6 & 0.446 \\
\hline Role as a parent & 26.5 & 46.1 & 18.4 & 35.4 & 14.2 & 28.9 & 0.004 \\
\hline Avoided or shunned by people & 40.7 & 2.2 & 34.1 & 5.1 & 25.4 & 1.5 & $<0.001$ \\
\hline \multicolumn{8}{|l|}{ Anticipated discrimination $(n=1080)^{b}$} \\
\hline Applying for a job & 48.2 & 38.6 & 40.6 & 35.0 & 27.2 & 27.4 & $<0.001$ \\
\hline Applying for education or training & 35.2 & 33.0 & 33.7 & 35.7 & 23.3 & 33.0 & 0.019 \\
\hline \multirow{2}{*}{\multicolumn{8}{|c|}{ Concealed or hidden mental health }} \\
\hline & & & & & & & \\
\hline problems & 77.7 & 0.8 & 69.0 & 0.6 & 62.4 & 1.1 & $<0.001$ \\
\hline
\end{tabular}

(a)

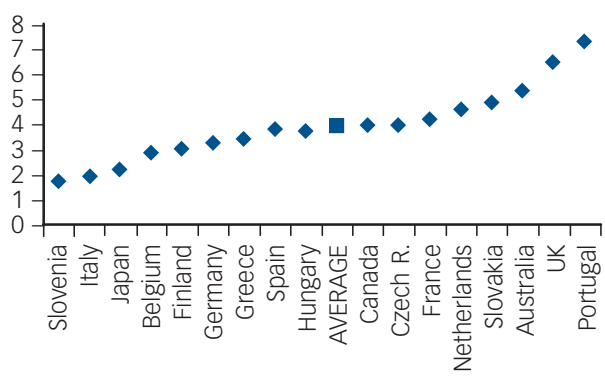

(b)

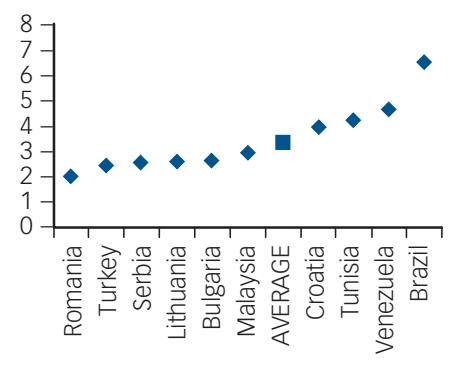

(c) 8

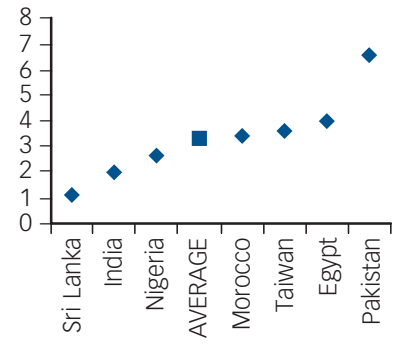

Fig. 1 Experienced discrimination scores by countries within groups with (a) very high, (b) high and (c) medium/low Human Development Index ( $n=1082)$.

Experienced discrimination: number of items (range 0-21) in which participants reported a disadvantage. Czech R., Czech Republic.
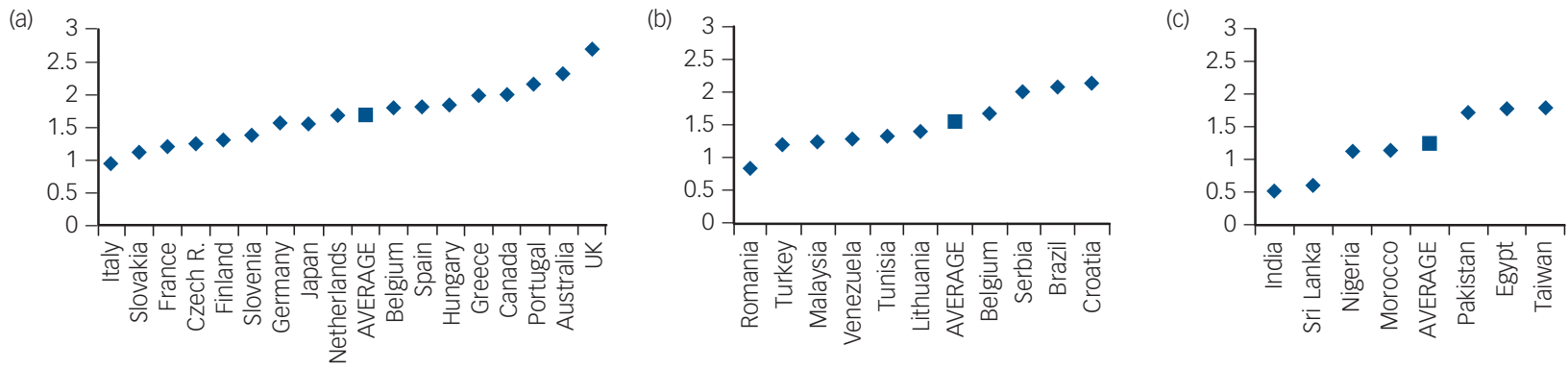

Fig. 2 Anticipated discrimination scores by countries within groups with (a) very high, (b) high and (c) medium/low Human Development Index $(n=1080)$.

Anticipated discrimination: number of items (range 0-4) in which participants reported a disadvantage. Czech R., Czech Republic. 
Table 3 Multilevel negative binomial regression models for experienced discrimination (overall sample $n=1082)^{a}$

\begin{tabular}{|c|c|c|c|c|c|c|}
\hline & \multicolumn{2}{|c|}{$M_{0}$ : variance components } & \multicolumn{2}{|c|}{$M_{1}: M_{0}+$ patient-level characteristics } & \multicolumn{2}{|c|}{$M_{2}: M_{1}+$ country-level characteristics } \\
\hline & Estimate (s.e.) & $P$ & Estimate (s.e.) & $P$ & Estimate (s.e.) & $P$ \\
\hline \multicolumn{7}{|l|}{ Fixed effects } \\
\hline Intercept & $1.25(0.07)$ & $<0.001$ & $0.88(0.16)$ & $<0.001$ & $0.90(0.18)$ & $<0.001$ \\
\hline \multicolumn{7}{|l|}{ Patient-level characteristics } \\
\hline Anticipated discrimination & & & $0.29(0.03)$ & $<0.001$ & $0.29(0.03)$ & $<0.001$ \\
\hline Age & & & $-0.01(0.00)$ & 0.001 & $-0.01(0.00)$ & 0.001 \\
\hline $\begin{array}{l}\text { Advantage to have a diagnosis of major } \\
\text { depressive disorder }\end{array}$ & & & $0.07(0.08)$ & 0.399 & $0.06(0.08)$ & 0.405 \\
\hline Ever admitted for psychiatric treatment & & & $0.32(0.07)$ & $<0.001$ & $0.32(0.07)$ & $<0.001$ \\
\hline $\begin{array}{l}\text { Six or more lifetime episodes } \\
\text { of depression }\end{array}$ & & & $0.23(0.07)$ & 0.001 & $0.23(0.07)$ & 0.001 \\
\hline \multicolumn{7}{|l|}{ Marital status } \\
\hline Married or co-habiting & & & Reference & & Reference & \\
\hline Single or no co-habiting partner & & & $0.01(0.08)$ & 0.970 & $0.01(0.08)$ & 0.984 \\
\hline Widowed, separated or divorced & & & $0.16(0.08)$ & 0.043 & $0.17(0.08)$ & 0.042 \\
\hline Low education & & & $-0.08(0.07)$ & 0.255 & $-0.08(0.07)$ & 0.230 \\
\hline \multirow{2}{*}{\multicolumn{7}{|c|}{$\begin{array}{l}\text { (up to secondary } \leqslant 16 \text { years) } \\
\text { Working condition }\end{array}$}} \\
\hline & & & & & & \\
\hline Full time or part time & & & Reference & & Reference & \\
\hline volunteer, sheltered or at home & & & $0.26(0.11)$ & 0.020 & $0.26(0.11)$ & 0.021 \\
\hline Unemployed or a student & & & $0.14(0.07)$ & 0.048 & $0.14(0.07)$ & 0.048 \\
\hline Retired & & & $-0.10(0.12)$ & 0.410 & $-0.10(0.12)$ & 0.420 \\
\hline \multicolumn{7}{|l|}{ Country-level characteristics, Human } \\
\hline \multicolumn{7}{|l|}{ Development Index (HDI) } \\
\hline Very high HDI & & & & & Reference & \\
\hline High HDI & & & & & $-0.10(0.15)$ & 0.531 \\
\hline Medium/low HDI & & & & & $0.01(0.17)$ & 0.941 \\
\hline \multicolumn{7}{|l|}{ Random-effect variances } \\
\hline Country-level & $0.13(0.04)$ & - & $0.09(0.03)$ & - & $0.09(0.03)$ & - \\
\hline $\begin{array}{l}\text { Proportion reduction in variance estimates } \\
\text { compared with intercept only model, \% }\end{array}$ & & & 30.8 & & 30.8 & \\
\hline Likelihood ratio test & 87.44 & $<0.001$ & 42.07 & $<0.001$ & 37.81 & $<0.001$ \\
\hline
\end{tabular}

Table 4 Multilevel poisson regression models for anticipated discrimination (overall sample $n=1080)^{\text {a }}$

\begin{tabular}{|c|c|c|c|c|c|c|}
\hline & \multicolumn{2}{|c|}{$M_{0}$ : variance components } & \multicolumn{2}{|c|}{$M_{1}: M_{0}+$ patient-level characteristics } & \multicolumn{2}{|c|}{$M_{2}: M_{1}+$ country-level characteristics } \\
\hline & Estimate (s.e.) & $P$ & Estimate (s.e.) & $P$ & Estimate (s.e.) & $P$ \\
\hline \multicolumn{7}{|l|}{ Fixed effects } \\
\hline Intercept & $0.39(0.06)$ & $<0.001$ & $0.30(0.15)$ & 0.043 & $0.42(0.16)$ & 0.008 \\
\hline \multicolumn{7}{|l|}{ Patient-level characteristics } \\
\hline Experienced discrimination & & & $0.06(0.01)$ & $<0.001$ & $0.06(0.01)$ & $<0.001$ \\
\hline Age & & & $-0.01(0.00)$ & 0.052 & $-0.01(0.00)$ & 0.052 \\
\hline $\begin{array}{l}\text { Advantage to have a diagnosis of major } \\
\text { depressive disorder }\end{array}$ & & & $0.07(0.07)$ & 0.356 & $0.07(0.07)$ & 0.374 \\
\hline $\begin{array}{l}\text { Six or more lifetime episodes } \\
\text { of depression } \\
\text { Marital status }\end{array}$ & & & $0.09(0.07)$ & 0.167 & $0.08(0.07)$ & 0.256 \\
\hline Married or co-habiting & & & Reference & & Reference & \\
\hline Single or no co-habiting partner & & & $0.16(0.08)$ & 0.040 & $0.15(0.08)$ & 0.054 \\
\hline Widowed, separated or divorced & & & $0.01(0.08)$ & 0.911 & $-0.01(0.08)$ & 0.994 \\
\hline \multicolumn{7}{|l|}{ Working condition } \\
\hline Full time or part time & & & Reference & & Reference & \\
\hline Volunteer, sheltered or at home & & & $-0.13(0.11)$ & 0.267 & $-0.08(0.12)$ & 0.473 \\
\hline Unemployed or a student & & & $0.01(0.07)$ & 0.976 & $0.01(0.07)$ & 0.989 \\
\hline Retired & & & $-0.16(0.12)$ & 0.201 & $-0.16(0.12)$ & 0.187 \\
\hline \multicolumn{7}{|l|}{ Country-level characteristics, Human } \\
\hline \multicolumn{7}{|l|}{ Development Index (HDI) } \\
\hline Very high $\mathrm{HDI}$ & & & & & Reference & \\
\hline High HDI & & & & & $-0.06(0.11)$ & 0.605 \\
\hline Medium/low HDI & & & & & $-0.34(0.13)$ & 0.007 \\
\hline \multicolumn{7}{|l|}{ Random-effect variances } \\
\hline Country level & $0.08(0.03)$ & - & $0.05(0.02)$ & - & $0.03(0.02)$ & - \\
\hline $\begin{array}{l}\text { Proportion reduction in variance } \\
\text { estimates compared with intercept only }\end{array}$ & & & & & & \\
\hline model, \% & & & 37.5 & & 62.5 & \\
\hline Likelihood ratio test & 88.45 & $<0.001$ & 21.86 & $<0.001$ & 13.30 & $<0.001$ \\
\hline
\end{tabular}


cross-level interaction was found between the significant individual-level experienced discrimination subscore and the country-level HDI classification.

\section{Discussion}

Reported discrimination among people with MDD is more severe in high-income countries. Multilevel regression models showed that this association was not significant for experienced discrimination, but significant for anticipated discrimination. This finding seems robust since the variation between countries decreased by $62.5 \%$ after adjusting for individual- and countrylevel variables and $25 \%$ of this decrease was explained by the HDI. The finding that anticipated discrimination differs across countries in parallel to their level of human development (with higher discrimination in the higher-income countries) deserves an in-depth conceptual analysis. In the DISC-12 anticipated discrimination occurs when a person limits their own involvement in important aspects of everyday life because of the expectation of being discriminated against (for example, when an individual does not apply for a job because he/she fully expects to fail in any such application). Therefore, respondents scoring high on the anticipated discrimination items not only anticipate discrimination, they also decided to give up and not pursue their goals - this is referred to as the 'why-try' effect. ${ }^{30}$ Thus, our findings indicate that anticipated discrimination has concrete consequences and a real impact on people's lives with more severe effect for those living in high-income countries.

\section{Interpretation of findings}

It therefore seems that the context (as a reflection of social norms and values that are typical of a given sociocultural group) matters in facilitating or hindering people with MDD to involve themselves in a number of important life activities. Other reasons for more anticipated discrimination in high-income countries may include the nature of employment, the broader socioeconomic context and the explanatory models of mental disorders and self-attribution. In this study almost twice as many patients living in high-income countries anticipated discrimination in the job domain, compared with those living in lower- and middle-income countries. The process of seeking entry (or re-entry) into the job market for anyone with a substantial disruption in work record in highly industrialised societies is typically frustrating and disheartening, and can be traumatic for recovering patients. ${ }^{31,32}$ In contrast, in traditional societies when patients recover from their symptoms, they are far more likely to find work and often find it much easier to reassume the work roles they had before. ${ }^{33}$ Further, in industrialised societies, the work environment is typically impersonal and can be intensely competitive. Thus, even when a person recovering from a severe episode of major depression finds a job, the profound sense of marginality and insecurity lingers on. Work relationships in industrialised countries are under more bureaucratic regimentation; this is less likely to be the case for patients living in traditional societies, since their work roles are more integrated with other aspects of their lives and are less likely to be taken away simply because of questions about their performance.

Another possible reason for lower levels of anticipated discrimination in low-income countries is the nature of community support. Most developing societies are based on collectivistic values, ${ }^{34}$ with primary emphasis on social relations and a range of conventions, rules and roles that tend to sustain long-term relationships and make isolation unusual even for people who are the most disabled. In contrast, in industrialised countries (where the social structure is generally individualistic) relationships are more likely to be bilaterally defined, contractual in nature and subject to constant re-evaluation and revocation. ${ }^{35}$ It is thus plausible that the intense individualism characteristic of some Western societies might not be conducive to recovery from mental ill health. Along with their emphasis on independence, self-reliance and personal freedom, individualistic value orientations also tend to foster fierce competition, frequent life changes and alienation, and they do not usually provide the kind of structured, stable and predictable environments that allow people with mental health conditions to recuperate at their own pace and to be reintegrated into society. There is initial empirical evidence that social context may make the difference for people with depression to perceive the 'others' as more or less supportive and/or stigmatising, since some recent studies found that greater community support and social capital are associated with less perceived discrimination. ${ }^{36,37}$

Explanatory models of mental disorders and self-attribution may also play a relevant role in shaping the perception of discrimination by people with depression. Traditional societies in low-income countries offer cultural belief systems that generally externalise causality of psychiatric problems (for example God's will, Karma or other supernatural entities), ${ }^{7,38}$ thus lessening individual and family blame. In contrast, in most high-income industrialised countries the prevailing paradigm is based on the biomedical model where mental disorders are assumed to have a biological basis. ${ }^{39}$ The notion that mental disorders are simply 'brain diseases' that exist as such in nature has proved to be extremely damaging to those with mental ill health. This notion is responsible for unwarranted and destructive pessimism about the chances of recovery, and has ignored what is actually going on in these people's lives, in their families and in the societies they live in. ${ }^{40}$ This results in stigmatisation and rejection from the outside, and self-attribution and self-blame from the inside. This is in contrast to low-income countries where expectations of severe mental disorders are that these conditions are like any other acute illness and societal reactions are in keeping with this view. ${ }^{41}$

In relation to the difference between high- and medium-/lowincome countries being particularly evident for anticipated discrimination, this may be because of a stronger and more visible service-user movement in some high-income countries campaigning on issues of discrimination, which has raised awareness among those with mental health problems of the discrimination they may experience. This is supported by a growing body of research detailing the nature and extent of discrimination across a range of settings. ${ }^{4}$ As service-user groups/movements emerge in lowincome countries, it may be that people with mental health problems in these countries will also become more aware of the discrimination they may experience.

\section{Strengths and limitations}

This study has the following strengths. The use of interviews to gather direct self-reports from people with depression, both of discrimination that was actually experienced (rather than hypothetical scenarios or vignettes) and that which was anticipated. Most research on discrimination and depression has largely been descriptive, concerning surveys of public attitudes about hypothetical situations rather than how discrimination is experienced by people with MDD. Moreover, collection of selfreport on discrimination may empower service users by giving them a voice and acknowledge the validity of their experience.

This study also has several limitations. Samples sizes in the participating countries were relatively small. Participants were selected from treated patients rather than from people with 
MDD living in the community, thus limiting the generalisability of results to all people with MDD living in participating sites. Selection bias could have occurred as participants were recruited on the basis of access to mental health services, the judgement of local research staff and their willingness to participate. Moreover, because of the relatively low sample size in the participating countries, we could not control for possible contextual differences within a given country (for example rural $v$. urban, deprived $v$. affluent areas). Disability and clinical severity measures were not used, therefore it was not easy to understand how far discrimination reported by respondents was more realistically attributable to disorder-related impairments or to negative appraisal of life circumstances influenced by current levels of depressive symptoms; this issue warrants further investigation. The cross-sectional study design does not provide evidence of causal relationships between putative predictors and levels of discrimination, nor about the time that it takes for stigma to develop and have consequences. The nature of this study was explanatory and therefore no a priori hypothesis was formulated about the direction of possible associations between cross-cultural differences and discrimination. No information was gathered on other possible minority statuses; this could have been a potential source of bias in case of patients with multiple minority statuses (for example ethnic, religious, sexual, physical illness). Finally, social desirability is a common limitation of self-report stigma measures that may vary cross-culturally.

\section{Implications for future research}

Overall, the results of this study suggest that close personal relationships and informal social support networks may play a significant role in buffering anticipated discrimination in people with depression; therefore the 'social distance' element of stigma is an important focus for future research. This also implies that culturally sensitive measures of social capital should be included in future cross-cultural research on stigma and discrimination. Moreover, we need more high-quality qualitative research in this field to gain a more in-depth insight about how discrimination really has an impact on peoples' lives and studies that will allow relating these reported/perceived findings to the outcome of mental disorders and the impact on people's lives. Longitudinal studies indicating how and when stigma develops and how stigma and its consequences change over time would also be very useful.

\section{A tentative agenda for future interventions}

The majority of people in high-income countries tend to attribute major depression to neurobiological causes. ${ }^{18}$ The percentage of the general public endorsing this view has steadily increased over the past 15 years, in parallel with the spread of the message launched by some campaigns worldwide claiming that mental illness is 'a disease like any other'. However, social distance and perceived danger associated with people with depression has not decreased significantly over the same period in Western Europe ${ }^{42}$ and the USA. ${ }^{43}$ Holding a neurobiological conception of mental disorders seems to increase the likelihood of support for treatment, but it is not associated with reduced stigmatisation beliefs; a neurobiological understanding of mental disorders seems to be rather associated with an increase of community rejection. ${ }^{18,39}$ The assumption underlying a number of anti-stigma campaigns launched over the past decades in high-income countries (i.e. educating people about the biological basis of mental disorders automatically leads to an improvement in their attitudes towards those with mental illness) therefore appears questionable. Because the public holds a tacit understanding of the aetiology of mental illness, our efforts need to move past this message. ${ }^{43}$ Reconfiguring stigma reduction strategies in highincome countries may require providers and advocates to shift to an emphasis on competence and inclusion. Efforts should prioritise inclusion, integration, competences for the reduction of cultural barriers to recognition, response and recovery. Unless we tackle stigma at the cultural level, the prospects for changing the lives of those affected by mental disorders will be unlikely to happen.

\section{Antonio Lasalvia, Md, PhD, U.O.C. Psichiatria, Azienda Ospedaliera Universitari Integrata (AOUI) di Verona, Verona, Italy; Tine Van Bortel, PhD, Cambridge Institute of Public Health, University of Cambridge, Cambridge, UK; Chiara Bonetto, PhD, Department of Public Health and Community Medicine, Section of Psychiatry, University of Verona, Verona, Italy; Geetha Jayaram, MD, Johns Hopkins University, schools of Medicine, Health Policy and Management and Armstrong Institute of Patient Safety, Baltimore, Maryland, USA; Jaap van Weeghel, PhD, Kenniscentrum Phrenos, Utrecht and TRANZO Department, Tilburg University, Tilburg, Netherlands; Silvia Zoppei, PhD, Department of Public Health and Community Medicine, Section of Psychiatry, University of Verona, Verona, Italy; Lee Knifton, Neil Quinn, Centre for Health Policy, University of Strathclyde, Glasgow, Scotland, UK; Kristian \\ Wahlbeck, MD, PhD, Finnish National Institute for Health and Welfare (THL), Helsinki, Finland; Doriana Cristofalo, Department of Public Health and Community Medicine, Section of Psychiatry, University of Verona, Verona, Italy; Mariangela Lanfredi PsyD, IRCCS Istituto Centro San Giovanni di Dio Fatebenefratelli, Brescia, Italy; Norman Sartorius, MD, MA, DPM, PhD, FRCPsych, Association for the Improvement of Mental Health Programmes, Geneva, Switzerland; Graham Thornicroft, PhD, Centre for Global Mental Health, Institute of Psychiatry, King's College, London, UK}

Correspondence: Antonio Lasalvia, U.O.C. Psichiatria, Azienda Ospedaliera Universitaria Integrata (AOUI), Policlinico "G.B. Rossi", P.le Scuro, 1037134 Verona, Italy. Email: antonio.lasalvia@univr.it

First received 2 Sep 2014, final revision 23 Dec 2014, accepted 15 Jan 2015

\section{Funding}

This paper arises from the Anti Stigma Programme European Network (ASPEN), which has received funding from the European Union in the framework of the Public Health Programme (EU Grant Agreement Number: 2007 301).

\section{Acknowledgements}

The ASPEN/INDIGO staff at coordinating centres: Graham Thornicroft, Tine Van Bortel, Samantha Treacy, Elaine Brohan, Shuntaro Ando, Diana Rose (King's College London, Institute of Psychiatry, London, England); Kristian Wahlbeck, Esa Aromaa, Johanna Nordmyr, Fredrica Nyqvist, Carolina Herberts (National Institute for Health and Welfare, Vasa, Finland); Oliver Lewis, Jasna Russo, Dorottya Karsay, Rea Maglajlic (Mental Disability Advocacy Centre, Budapest, Hungary); Antonio Lasalvia, Silvia Zoppei, Doriana Cristofalo, Chiara Bonetto (Department of Public Health and Community Medicine, Section of Psychiatry, University of Verona, Italy); Isabella Goldie, Lee Knifton, Neil Quinn (Mental Health Foundation, Glasgow, Scotland); Norman Sartorius (Association for the improvement Health Foundation, Glasgow, Scotland); Norman Sartorius (Assoc
of mental health programmes (AMH), Geneva, Switzerland).

The ASPEN/INDIGO staff at partner centres: Chantal Van Audenhove, Gert Scheerder, Else Tambuyzer (Katholieke Universiteit Leuven, Belgium); Valentina Hristakeva, Dimitar Germanov (Global Initiative on Psychiatry Sofia, Bulgaria); Jean Luc Roelandt, Simon Vasseur Bacle, Nicolas Daumerie, Aude Caria (Etablissement Public Santé Mentale Lille-Métropole (EPSM/CCOMS), France): Harald Zaske, Wolfgang Gaebel (Heinrich-Heine Universitat Dusseldorf, Rheinische Kliniken Dusseldorf, Germany); Marina Economou, Eleni Louki, Lily Dusseldorf, Rheinische Kliniken Dusseldorf, Germany); Marina EConomou, Eleni LoukI, Lily
Peppou, Klio Geroulanou (University Mental Health Institute (UMHRI (EPIPSI), Greece); Judit Harangozo, Julia Sebes, Gabor Csukly (Awakenings Foundation, Hungary); Giuseppe Rossi, Mariangela Lanfredi, Laura Pedrini (IRCCS Istituto Centro San Giovanni di Dio Fatebenefratelli, Brescia, Italy; Arunas Germanavicius, Natalja Markovskaja, vytis Valantinas (Vilnius University, Lithuania); Jaap van Weeghel, Jenny Boumans, Eleonoor Willemsen, Annette Plooy (Stichting Kenniscentrum Phrenos (KCP), The Netherlands): Teresa Duarte, Fatima Jorge Monteiro (Associação para o Estudo e Integração Psicossocial, Portugal); Radu Fatima Jorge Monteiro (Associação para o Estudo e Integração Psicossocial, Portugal), Radu
Teodorescu, Iuliana Radu, Elena Pana (Asociatia din Romania de Psihiatrie Comunitara, Teodorescu, Iuliana Radu, Elena Pana (Asociatia din Romania de Psihiatrie Comunitara,
Romania; Janka Hurova, Dita Leczova (Association for Mental Health INTEGRA, o. Z., Slovakia); Vesna Svab, Nina Konecnik (University Psychiatric Hospital, Slovenia); Blanca Reneses, Juan J Lopez-Ibor, Nerea Palomares, Camila Bayon (Instituto de Psiquiatria at the Hospital Universitario San Carlos, Spain); Alp Ucok, Gulsah Karaday (Foundation of Psychiatry Clinic of Medical Faculty of Istanbul (PAP), Turkey); Nicholas Glozier, Nicole Cockayne (Brain \& Mind Research Institute, Sydney Medical School, University of Sydney, Australia); Luís Fernando Tófoli, Maria Suely Alves Costa (Universidade Federal do Ceará, Campus Sobral, Brazil); Roumen Milev, Teresa Garrah, Liane Tackaberry, Heather Stuart (Department of Psychiatry, Queen's University, Canada/Providence Care, Mental Health Services, Kingston, Ontario, Canada; Branka Aukst Margetic, Petra Folnegovic Groiæ (Department of Psychiatry, University Hospital Centre ZagrebMiro Jakovljeviæ, Croatia): Barbora Wenigová, elepová Pavla (Centre for Mental Health Care Development, Prague, Czech Republic); Doaa Nader Radwan (Institute of Psychiatry, Ain Shams University, Cairo, Egypt); Pradeep Johnson, Ramakrishna Goud, Nandesh, Geetha Jayaram (St. John's Medical Egypt); Pradeep Johnson, Ramakrishna Goud, Nandesh, Geetha Jayaram (St. John's Medical
College Hospital, St John's National Academy of Health Sciences, Bangalore, India; Shuntaro Ando (Social Psychiatry, Tokyo Metropolitan Institute of Medical Science, Tokyo, Japan; 
Yuriko Suzuki, Tsuyoshi Akiyama, Asami Matsunaga, Peter Bernick (NTT Kanto Hospital Japan); Bawo James (Federal Neuropsychiatric Hospital, USELU, Benin City, Nigeria; Bolanle Ola, Olugbenga Owoeye (Federal Neuropsychiatric Hospital Yaba, Lagos, Nigeria): Yewande Oshodi (Department of Psychiatry, College of Medicine University of Lagos and Lagos Hospital Maiduguri, Nigeria); Kok-Yoon Chee, Noti Ali (Kuala Lumpur Hospital and Selayang Hospital, Malaysia); Nadia Kadri, Dounia Belghazi, Yassine Anwar (Ibn Rushd University Psychiatric Centre, Casablanca, Morocco); Nashi Khan, Rukhsana Kausa (University of the Punjab, Department of Applied Psychology and Centre for Clinical Psychology, Lahore, Pakistan); IVona Milacic Vidojevic (Faculty for Special Education and Rehabilitation, Belgrade, Serbia); Athula Sumathipala (Institute of Psychiatry, King's College ondon/Institute for Research and Development, Sri Lanka); Chih-Cheng Chang (Chi Mei Ouali, Haye Ouertani, Rabaa Jomli, Abdelhafidh Ouertani, Khadija Kaaniche (Razi Hospital Manouba, Department of Psychiatry, Tunis, Tunisia); Ricardo Bello, Manuel Ortega, Arturo Melone María Andreína Marques, Francisco Marco, Arturo Ríos, Ernesto Rodríguez, Arianna Laguado (Hospital Universitario de Caracas, Caracas, Venezuela). G.T. is supported by the National Institute for Health Research (NIHR) Collaboration for Leadership in Applied Health Research and Care South London at King's College London Foundation Trust. The views expressed are those of the author(s) and not necessarily those of the NHS, the NIHR or the Department of Health. G.T. acknowledges financial support from the Department of Health via the NIHR Biomedical Research Centre and Dementia Unit awarded to South London and Maudsley NHS Foundation Trust in partnership with King's College London and King's College Hospital NHS Foundation Trust. G.T. is supported by the European Union Seventh Framework Programme (FP7/2007-2013) Emerald project.

\section{References}

1 Murray CJL, Vos T, Lozano R, Naghavi M, Flaxman AD, Michaud C, et al. Disability-adjusted life years (DALYS) for 291 diseases and injuries in 21 regions, 1990-2010: a systematic analysis for the Global Burden of Disease Study 2010. Lancet 2012; 380: 2197-223.

2 Kohn R, Saxena S, Levav I, Saraceno B. The treatment gap in mental health care. Bull World Health Organ 2004; 82: 858-66.

3 Andrade LH, Alonso J, Mneimneh Z, Wells JE, Al-Hamzawi A, Borges G, et al. Barriers to mental health treatment: results from the WHO World Mental Health surveys. Psychol Med 2013; 9: 1-15.

4 Thornicroft G. Shunned: Discrimination Against People with Mental Illness. Oxford University Press, 2006.

5 Kohrt BA, Hruschka DJ. Nepali concepts of psychological trauma: the role of idioms of distress, ethnopsychology and ethnophysiology in alleviating suffering and preventing stigma. Cult Med Psychiatry 2010; 34: 322-52.

6 Quinn N, Knifton L. Beliefs, stigma and discrimination associated with mental health problems in Uganda: implications for theory and practice. Int J SOC Psychiatry 2014; 60: 554-61.

7 Patel V. Explanatory models of mental illness in sub-Saharan Africa. Soc Sci Med 1995; 40: 1291-8.

8 Carpenter-Song E, Chu E, Drake RE, Ritsema M, Smith B, Alverson H. Ethno-cultural variations in the experience and meaning of mental illness and treatment: implications for access and utilization. Transcult Psychiatry 2010; 47: 224-51.

9 Sanchez F, Gaw A. Mental health care of Filipino Americans. Psychiatr Serv 2007; 58: 810-5.

10 Ting JY, Hwang WC. Cultural influences on help-seeking attitudes in Asian American students. Am J Orthopsychiatry 2009; 79: 125-32.

11 Vega WA, Rodriguez MA, Ang A. Addressing stigma of depression in Latino primary care patients. Gen Hosp Psychiatry 2010; 32: 182-91.

12 Knifton L. Understanding and addressing the stigma of mental illness with ethnic minority communities. Health Sociol Rev 2012; 21: 287-98.

13 Assefa D, Shibre T, Asher L, Fekadu A. Internalized stigma among patients with schizophrenia in Ethiopia: a cross-sectional facility-based study. BMC Psychiatry 2012; 12: 239

14 Sharaf AY, Ossman LH, Lachine OA. A cross-sectional study of the relationships between illness insight, internalized stigma, and suicide risk in individuals with schizophrenia. Int J Nurs Stud 2012; 49: 1512-20.

15 Adewuya AO, Owoeye AO, Erinfolami AO, Ola BA. Correlates of self-stigma among outpatients with mental illness in Lagos, Nigeria. Int J Soc Psychiatry 2011; 57: 418-27.

16 Sorsdahl KR, Stein DJ. Knowledge of and stigma associated with mental disorders in a South African community sample. J Nerv Ment Dis 2010; 198 742-7.

17 Angermeyer MC, Dietrich S. Public beliefs about and attitudes towards people with mental illness: a review of population studies. Acta Psychiatr Scand 2006; 113: 163-79.

18 Pescosolido BA, Martin JK, Long JS, Medina TR, Phelan JC, Link BG. "A disease like any other"? A decade of change in public reactions to schizophrenia, depression, and alcohol dependence. Am J Psychiatry 2010; 167: $1321-30$
19 Tanaka G, Inadomi H, Kikuchi Y, Ohta Y. Evaluating community attitudes to people with schizophrenia and mental disorders using a case vignette method. Psychiatry Clin Neurosci 2005; 59: 96-101.

20 de Toledo Piza Peluso E, de Araújo Peres C, Luís Blay S. Public conceptions of schizophrenia in urban Brazil: symptom identification and causal attributions. Soc Psychiatry Psychiatr Epidemiol 2008; 43: 792-9.

21 Oshodi YO, Abdulmalik J, Ola B, James BO, Bonetto C, Cristofalo D, et al. Pattern of experienced and anticipated discrimination among people with depression in Nigeria: a cross-sectional study. Soc Psychiatry Psychiatr Epidemiol 2014; 49: 259-66.

22 Alonso J, Buron A, Rojas-Farreras, de Graaf R, Haro JM, de Girolamo G, et al. Perceived stigma among individuals with common mental disorders. J Affect Disord 2009; 118: 180-6.

23 Brohan E, Gauci D, Sartorius N, Thornicroft G. Self-stigma, empowerment and perceived discrimination among people with bipolar disorder or depression in 13 European countries: The GAMIAN-Europe study. J Affect Disord 2011; 129: $56-63$.

24 Krajewski C, Burazeri G, Brand H. Self-stigma, perceived discrimination and empowerment among people with a mental illness in six countries: Pan European stigma study. Psychiatry Res 2013; 210: 1136-46.

25 Lasalvia A, Zoppei S, Van Bortel T, Bonetto C, Cristofalo D, Wahlbeck K, et al. Global pattern of experienced and anticipated discrimination reported by people with major depressive disorder: a cross-sectional survey. Lancet 2013; 381: 55-62.

26 Thornicroft G, Brohan E, Rose D, Sartorius N, Leese M. Global pattern of experienced and anticipated discrimination against people with schizophrenia: a cross-sectional survey. Lancet 2009; 373: 408-15.

27 American Psychiatric Association. Diagnostic and Statistical Manual of Mental Disorders (4th edn, text revision) (DSM-IV-TR). APA, 2000.

28 Brohan $E$, Clement $S$, Rose $D$, Sartorius N, Slade M, Thornicroft G. Development and psychometric evaluation of the Discrimination and Stigma Scale (DISC). Psychiatry Res 2013; 208: 33-40.

29 United Nations Development Programme (UNDP). Human Development Report 2010. The Real Wealth of Nations: Pathways to Human Development. UNPD, 2010.

30 Corrigan PW, Larson JE, Rüsch N. Self-stigma and the "why try" effect: Impact on life goals and evidence-based practices. World Psychiatry 2009; 8 75-81.

31 Boardman J, Grove B, Perkins R, Shepherd G. Work and employment for people with psychiatric disabilities. Br J Psychiatry 2003; 182: 467-8.

32 Stuart $\mathrm{H}$. Mental illness and employment discrimination. Curr Opin Psychiatry 2006; 19: 522-6.

33 Warner R. Recovery from Schizophrenia: Psychiatry and Political Economy (3rd edn). Brunner-Routledge, 2004.

34 Hofstede G. Cultures Consequences. International Differences in Work Related Values. Sage, 1980.

35 Papadopoulos C, Foster J, Caldwell K. 'Individualism-collectivism' as an explanatory device for mental illness stigma. Community Ment Health J 2013; 49: $270-80$.

36 Webber M, Corker E, Hamilton S, Weeks C, Pinfold V, Rose D, et al. Discrimination against people with severe mental illness and their access to social capital: findings from the Viewpoint survey. Epidemiol Psychiatr Sci 2014; 23: 155-65.

37 Zoppei S, Lasalvia A, Bonetto C, Van Bortel T, Nyqvist F, Webber M, et al. Social capital and reported discrimination among people with depression in 15 European countries. Soc Psychiatry Psychiatr Epidemiol 2014; 49: 1589-98.

38 Burnard P, Naiyapatana W, Lloyd G. Views of mental illness and mental health care in Thailand: a report of an ethnographic study. J Psychiatr Ment Health Nurs 2006; 13: 742-9.

39 Angermeyer MC, Daubmann A, Wegscheider K, Mnich E, Schomerus G, Knesebeck OV. The relationship between biogenetic attributions and desire for social distance from persons with schizophrenia and major depression revisited. Epidemiol Psychiatr Sci 2014; 30: 1-7.

40 Read J. Why promoting biological ideology increases prejudice against people labelled 'schizophrenic'. Aust Psychol 2007; 42: 118-28.

41 Waxier NE. Is mental illness cured in traditional societies? A theoretical analysis. Cult Med Psychiatry 1977; 1: 233-53.

42 Angermeyer MC, Holzinger A, Matschinger $\mathrm{H}$. Mental health literacy and attitude towards people with mental illness: a trend analysis based on population surveys in the eastern part of Germany. Eur Psychiatry 2009; 24: $225-32$

43 Pescosolido BA, Medina TR, Martin JK, Long JS. The "backbone" of stigma: identifying the global core of public prejudice associated with mental illness. Am J Public Health 2013; 103: 853-60. 